\title{
Altered collagen metabolism and delayed healing in a novel model of ischemic wounds
}

\author{
DANIEL A. SCHWARZ, MDa; WILLIAM J. LINDBLAD, PhD'; RILEY S. REES, MD
}

\begin{abstract}
Cellular mechanisms occurring in the healing wound have been well described in various animal models. However. the events associated with wound healing seen in ischemic skin have not been as thoroughly defined. In this series of experiments, we created a novel model of excisional skin wounds under gradient ischemia to study the cellular and extracellular events leading to delayed healing. We hypothesized that altered collagen metabolism accounts for delayed wound healing in ischemic skin. Three pairs of $4 \mathrm{~mm}$ punch wounds were made 4 days after bipedicle skin flaps were created on the dorsum of rats. Sham-operated control animals had the same punch wounds without flap creation. The kinetics of excisional wound healing were measured by means of computerized planimetry. In addition, wounds were excised with a $6 \mathrm{~mm}$ trephine, radiolabelled with $\left({ }^{3} \mathrm{H}\right)$-proline and in vitro collagen synthesis determined as collagenase digestible protein along with quantitation of DNA content. Total collagen deposition was determined as 4-hydroxy-L-proline by high-performance liquid chromatography, and wounds were histologically evaluated. Data was analyzed by means of two-way analysis of variance. Although control wounds healed by day 10, flap wounds consistently had greater surface area on days 2, 4, 6, 8, and 12 ( $P<0.001)$. Relative collagen synthesis (\% collagen/noncollagen protein), as measured by an in vitro synthesis method, showed no statistically significant differences between flap and controls wounds. However, the total collagen content (deposition) as measured by 4-hydroxy-L-proline was significantly lower in flap wounds compared with controls on days $7(p<0.05)$ and $9(p<0.001)$. In addition, a significant increase occurred in DNA content in the flap wounds on days $7(p<0.05)$ and $9(p<0.001)$ versus control wounds. These data indicate that, in ischemic wounds, significantly less collagen is deposited despite the inherent ability of the tissue to synthesize appropriate levels of collagen. Because the in vitro collagen synthesis technique only assesses the ability of the tissue to synthesize collagen in a well oxygenated environment, one cannot be assured that the tissue expresses this potential in vivo. However, these data are consistent with the hypothesis that the delay in wound closure is due to an alteration in collagen metabolism which results in a net decrease in collagen accumulation. Because of the observed increase in DNA within the ischemic wounds, we suggest that there is prolonged inflammation in these wounds which may enhance collagen degradation through the release of proteases. In addition, there may be an inability of the tissue to maintain appropriate levels of collagen in this inflammatory wound environment. (WOUND REP REG 1995;3:204-12)
\end{abstract}

Several animal models have been used to study different cellular and biochemical processes of wound healing. Examples include the Seyle "granuloma" pouch to study angiogenesis and effects of radiation, ${ }^{1}$ along

From the Section of Plastic and Reconstructive Surgery, Department of Surgery, University of Michigan, Ann Arbor: and Department of Pharmaceutical Sciences, Wayne State University, Detroit, Mich.

Reprint requests: Riley S. Rees, MD. Section of Plastic and Reconstructive Surgery, The University of Michigan, 1500 East Medical Center Drive. Ann Arbor, MI 48109-0340.

Copyright (C) 1995 by The Wound Healing Society.

1067-1927/95 $\$ 3.00+036 / 1 / 66036$

\begin{tabular}{|ll|}
\hline 4-Hyp & 4-Hydroxy-L-proline \\
TCA & Trichloroacetic acid \\
\hline
\end{tabular}

with the rabbit ear chamber to study the effects of antiseptics and leukocyte migration in a healing wound. ${ }^{2,3}$ Eriksson's hairless mouse ear model is a simple and inexpensive way to study microcirculation. ${ }^{4}$ Several refinements and interventions by Barker et al. ${ }^{5,6}$ and others have further defined the pathophysiology of microvascular changes seen in Eriksson's model. Implantable devices have been used in rat and rabbit skin incisions to study the effects of oxygen on wound healing. ${ }^{7,8}$ The rabbit cornea model has been 
used to study the effects of fibrin, activated platelets, and activated macrophages on angiogenesis and collagen synthesis. ${ }^{9,10}$ The rabbit ear has also been used to study microvascular changes and associated wound healing, both in regards to ischemia and testing of growth factors to promote healing. ${ }^{11}$ Although several parameters of microvascular changes and rates of wound healing have been assessed, no model has been able to reproduce the changes seen in healing wounds with gradient ischemia.

We have modified $^{12}$ a bipedicle skin flap as previously described on the rat dorsum. ${ }^{13}$ Our previous studies suggest that the ischemic effects seen in these flaps may be attributed to an association between inflammatory cells, oxidants, and the degree of ischemia. ${ }^{12,14,15}$ In this study, we present a novel model of wound healing that combines a series of excisional punch wounds within these flaps to study the effects of gradient ischemia on collagen metabolism and delayed wound healing. This model can assess differences in wound healing by several parameters. It helps to define the cellular events occurring in ischemic wounds by correlating measures of collagen metabolism, kinetics of wound healing, histologic characteristics, and DNA content with delayed healing.

\section{MATERIALS AND METHODS}

\section{Animal Model}

All experiments were in accord with the standards in "Guide for the Care and Use of Laboratory Animals," prepared by the Institute of Laboratory Animal Resources and published by the National Institutes of Health (NIH Publication No. 86-23, Revised 1985), and supervised by veterinarians from the Unit for Laboratory Animal Care of the University of Michigan Medical at the Veterans Administration Medical Center, Ann Ärbor, Michigan.

Sprague-Dawley rats (150 to 200 gm; Charles River Laboratories, Portage, Mich.) were anesthetized with intramuscular ketamine hydrochloride $(100 \mathrm{mg} / \mathrm{kg}$ body weight; Parke Davis, Morris Plains, N.J.). The dorsal hair was clipped, and a $10 \times 4 \mathrm{~cm}$ clear rectangular template was used to outline the area of study on the back. Under sterile conditions, the bipedicle flaps were outlined with a surgical marker and cut through the panniculus carnosus, preserving the base at the iliac crest and the tip at the scapula by means of our modification ${ }^{12}$ of the technique described by McFarlane et al. ${ }^{13}$ (Figure $1, A$ ). The flaps were then
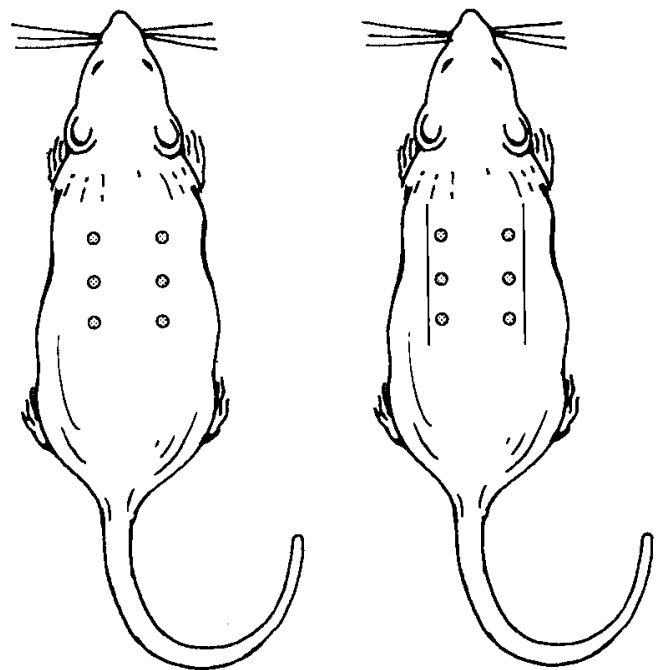

Control

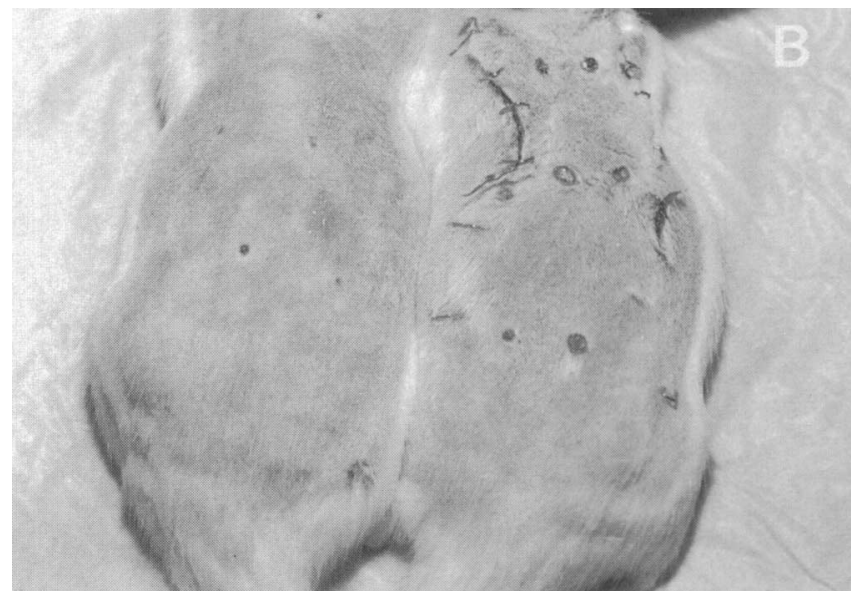

Figure 1 A, Illustration of our surgical models. Circles show approximate location of the three pairs of $4 \mathrm{~mm}$ punch wounds created in the skin on the rat's dorsum. Sham-operated control (leff) consists of punch wounds alone. The parallel lines on the bipedicle fiap icon (righf) represent incisions from the skin through the panniculus carnosus used to create the flap. B, Photograph of sham-operated control (leff) and flap wound animai (right) 8 days after punch wounds shows the wound area defects. The flap wounds appear much larger, whereas most of the control wounds have healed.

immediately secured with surgical staples (Richard Allen Medical Inc, Richland, Mich.).

A $4 \mathrm{~mm}$ Baker punch (Baker/Cummins, Miami, Fla.) fastened into a $3 / 8$ " electric drill was used to create the excisional wounds 4 days after the flaps were created. With sterile technique, the bipedicle skin flaps on the rats were grasped between both index fingers and thumbs. The folded skin flap was placed flush against a cutting board and two punches were drilled through the folded skin at three sites on the flap: one over the iliac crest, a second in the midflap, and the third over the scapula. Care was taken to remove the skin punch from the wound. Power 
equipment was necessary to make the punches in the bipedicle flap because the skin was thicker and less pliable than the nonischemic skin. A similar procedure was performed on the sham-operated control animals at the same locations (Figure $1, A$ ). The animals were returned to their cages and received food and water ad libitum.

\section{Measurement of excisional wound healing}

Total area of skin wounds was measured every other day with the NIH Image 1.44 computer analysis program." The wound area was traced onto transparency film (Labelon Corp., Canadaigua, N.Y.) with a fine marker. The transparency film was photocopied onto plain paper and subsequently scanned into a pic-format file with the Lightning Scan Pro 256 (Thunderware, Orinda, Calif.) hand scanner. Tissue area was calculated with nonrectangular area analysis functions used by the Image 1.44 software, and area measurements were expressed in square millimeters as mean \pm standard deviation of the mean. Repeated measurements were made every other day through day 12 .

\section{Experimental design}

The study comprised two groups of rats sampled at 1 , $3,5,7$, and 9 days after creation of punch wounds. In one group, excisional punch wounds were made in bipedicle skin flaps as described, and the second group was the sham-operated control. Each group consisted of a minimum of seven animals per time interval $(n=$ 4 flaps and $n=3$ controls). Animals were killed with an overdose of pentobarbital ( $200 \mathrm{mg} / \mathrm{kg}$ body weight), the skin flap (or corresponding area on sham controls) was removed, and excisional wound samples were immediately taken with a $6 \mathrm{~mm}$ trephine. Tissue was assayed for 4-hydroxy-L-proline (4-Hyp) content, collagen synthesis, DNA content, and histologic evaluation. A minimum of one excisional wound per animal was used for eäch 4-Hyp determination and histologic analysis, whereas two wounds were required per animal at each time interval for the collagen synthesis and DNA assays.

\footnotetext{
* NIH Image 1.44, is a public domain image processing and analysis program that can acquire, display, enhance, analyze, and animate images from various file formats. NIH Image 1.44 was written by Wayne Rasband at the National Institutes of Health Research Services Branch and was obtained through the University of Michigan School of Engineering. For these studies, Image 1.44 was employed on a Macintosh IICX with 5 MB RAM memory and AppleColor High Resolution RGB monitor (Apple Computer, Inc., Cupertino, Calif.).
}

\section{Histologic evaluation}

Excised wounds were immediately bisected and placed in $10 \%$ buffered formalin then imbedded in paraffin. Sections $(5 \mu \mathrm{m})$ were cut, mounted, and stained with hematoxylin and eosin and Masson's trichrome.

\section{4-Hyp determination}

Total collagen content of tissue was determined by quantitation of 4-Hyp by an high-performance liquid chromatography method. ${ }^{16}$ In brief, wound tissue was minced and placed into borosilicate screw cap culture tubes containing $5 \mathrm{ml}$ of $6 \mathrm{~N} \mathrm{HCl}$. The tubes were securely sealed and heated for 16 hours at $121^{\circ} \mathrm{C}$. The resulting hydrolysates were filtered through glass wool, evaporated to dryness, and reconstituted in $400 \mu \mathrm{l}$ of water. Twenty microliter aliquots were analyzed by highperformance liquid chromatography after pre-column derivatization with 7-chloro-4-nitrobenzo-2-oxa-1,3 diazole chloride to produce a chromophore. Quantitation was based on an area ratio with an internal standard of cis 4-Hyp and values expressed as nanomoles.

\section{Collagen synthesis}

Collagen synthesis in tissues was determined after the metabolic labeling of total proteins with $\left[{ }^{3} \mathrm{H}\right]$-proline. ${ }^{17}$ Two $6 \mathrm{~mm}$ excisional wounds per animal were minced and incubated with $10 \mu \mathrm{Ci}$ of $\left[{ }^{3} \mathrm{H}\right]$-proline (L$\left[5-{ }^{3} \mathrm{H}\right]$-proline, $28 \mathrm{Ci} / \mathrm{mmol}$; Amersham Corp., Arlington Heights, Ill.) in $3 \mathrm{ml}$ of Hank's balanced salt solution at $37^{\circ} \mathrm{C}$ for 4 hours. This procedure was followed by a 10 -minute incubation at $90^{\circ} \mathrm{C}$ to denature any proteases: The samples were homogenized, chilled, and protein precipitated by the addition of cold 50\% trichloroacetic acid (TCA) to a final concentration of $5 \%$. The precipitates were washed four times in cold 5\% TCA to remove unincorporated ${ }^{3} \mathrm{H}$ proline. In addition, the samples were digested with RNase ( $20 \mu \mathrm{g} / \mathrm{ml}$; Sigma Chemical Co., St. Louis, Mo.) for 5 minutes to remove any transfer RNA which may have incorporated ${ }^{3} \mathrm{H}$-proline and reprecipitated with $5 \%$ TCA. The samples were then solubilized in $0.2 \mathrm{~N}$ $\mathrm{NaOH}$ and divided into three fractions, one of which was digested with highly purified bacterial collagenase (Worthington Biochemical Corp., Freehold, N.J.) for 3 hours at $37^{\circ} \mathrm{C}$. Undigested proteins were reprecipitated by the addition of TCA-tannic acid. Radioactivity in the solubilized collagen and in the precipitated noncollagen proteins was then measured with liquid scintillation spectrometry. The incorporation of ${ }^{3} \mathrm{H}$-proline into collagen as compared with noncollagen protein was calculated taking into account the imino acid enrichment in collagen. ${ }^{18}$ 


\section{DNA content}

DNA content was measured fluorometrically from samples obtained during the collagen synthesis assay, after being solubilized in $0.2 \mathrm{~N} \mathrm{NaOH}$. Values were determined in duplicate and expressed in nanograms.

\section{Data analysis}

The main effect variables in this study were the following: (1) bipedicle flap with excisional punch wounds, (2) sham-operated controls with excisional punch wounds, and (3) kinetics of wound healing (every other day from 1 through 9 except for wound area measurements which were even days from 0 through 12). Each group consisted of a minimum of three animals. In general, repeated-measures analysis of variance of various designs were used to detect between group differences and interactions among main effect variables for each outcome variable measured. Post hoc pair-wise comparisons of individual means were performed if the overall analysis of variance showed a significant $\mathrm{F}$ ratio. Pair-wise comparisons were made with $t$ comparison of least square means with appropriate adjustment of $p$ value for multiple comparisons. Alpha error was set at 0.05 , and beta error was set at 0.20 .

\section{RESULTS}

Measurement of the wound defect present after excisional wounding showed considerable differences between flap and control wounds. Figure 1, $B$, presents a photograph taken of wounds after 8 days. The gross difference in size can be seen by a larger defect present in the ischemic wounds. Absolute wound area was calculated for control and flap wounds by computer imaging for days $0,2,4,8,10$, and 12 (Figure 2). Overall, there was a persistent failure of the flap wound area to decrease throughout the 12 days of study, with the size remaining essentially the same. In contrast, the control wounds showed a decrease in the wound size with complete healing by day 10 . This delay in healing as measured by persistence of wound defect was statistically significant on days 2 through 12 (except day 10).

Histologic examination of sections of wounds from days 1 and 3 failed to show qualitative differences between ischemic and control wounds. All had a large amount of eschar with underlying inflammatory cells and cellular debris with minimal new extracellular matrix. By day 5, evidence of extracellular matrix deposition and re-epithelization in the controls on both hematoxylin and eosin and trichrome staining was apparent. The flap wounds were associated with a

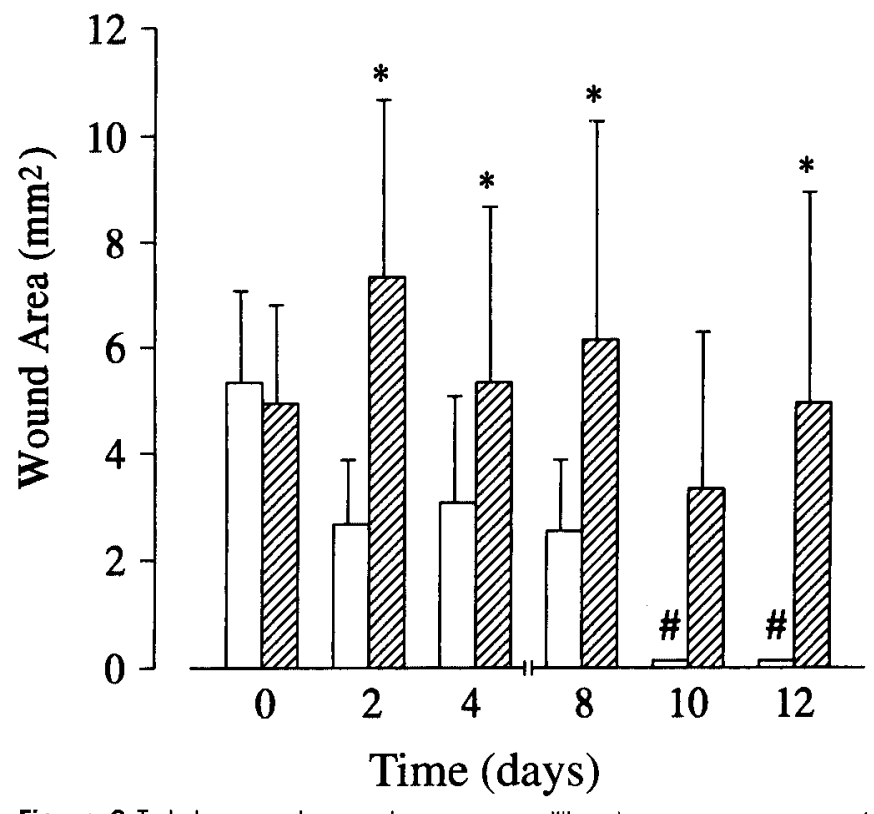

Figure 2 Total wound area in square millimeters are compared between flap (slashed bars) and control (open bars) wounds eveny other day from day o through 12. A persistent defect or excisional area reflecting a delay in healing occurs in wounds from bipedicle flaps $(p<0.001)$. After 8 days, the control wounds have healed $\left({ }^{*}\right)$.

persistence of clot with neutrophils and erythrocytes overlying the wound. Both groups showed evidence of granulation tissue formation with an apparent greater number of blood vessels noted in the control samples than in flaps. Day 7 showed a persistence of clot above a newly formed but thin layer of epithelium in the flap wounds. In addition there were erythrocyte and inflammatory cells immediately beneath the epithelia (Figure 3, A). The control wounds show a solid layer of epithelia above, underlying fibroblasts and extracellular matrix. Trichrome staining showed lightly stained scattered areas of new collagen fibrils in the control samples (Figure 3, E). Day 9 sections of flap wounds (hematoxylin and eosin and trichrome staining) showed a thin layer of deeply stained collagen densely populated with fibroblasts and comprising a larger wound area than the controls. The control wounds had a well formed layer of epithelia with underlying fibroblasts and a significant amount of lightly stained collagen fibers. Overall, the flap wounds appeared to be much thinner from epithelium to the panniculus carnosus and were deficient in lighter staining new collagen deposition (Figure $3, E, F$ ).

The amount of 4-Hyp was analyzed as a measure of total collagen content in wounds on days $1,3,5,7$, and 9 (Figure 4). Days 1, 3, and 5 showed similar results, with control levels beginning at $1006 \mathrm{nmol} /$ sample then leveling in the $1200 \mathrm{nmol} / \mathrm{sample}$ range. The flap wounds started lower at $813 \mathrm{nmol} / \mathrm{sample}$, 

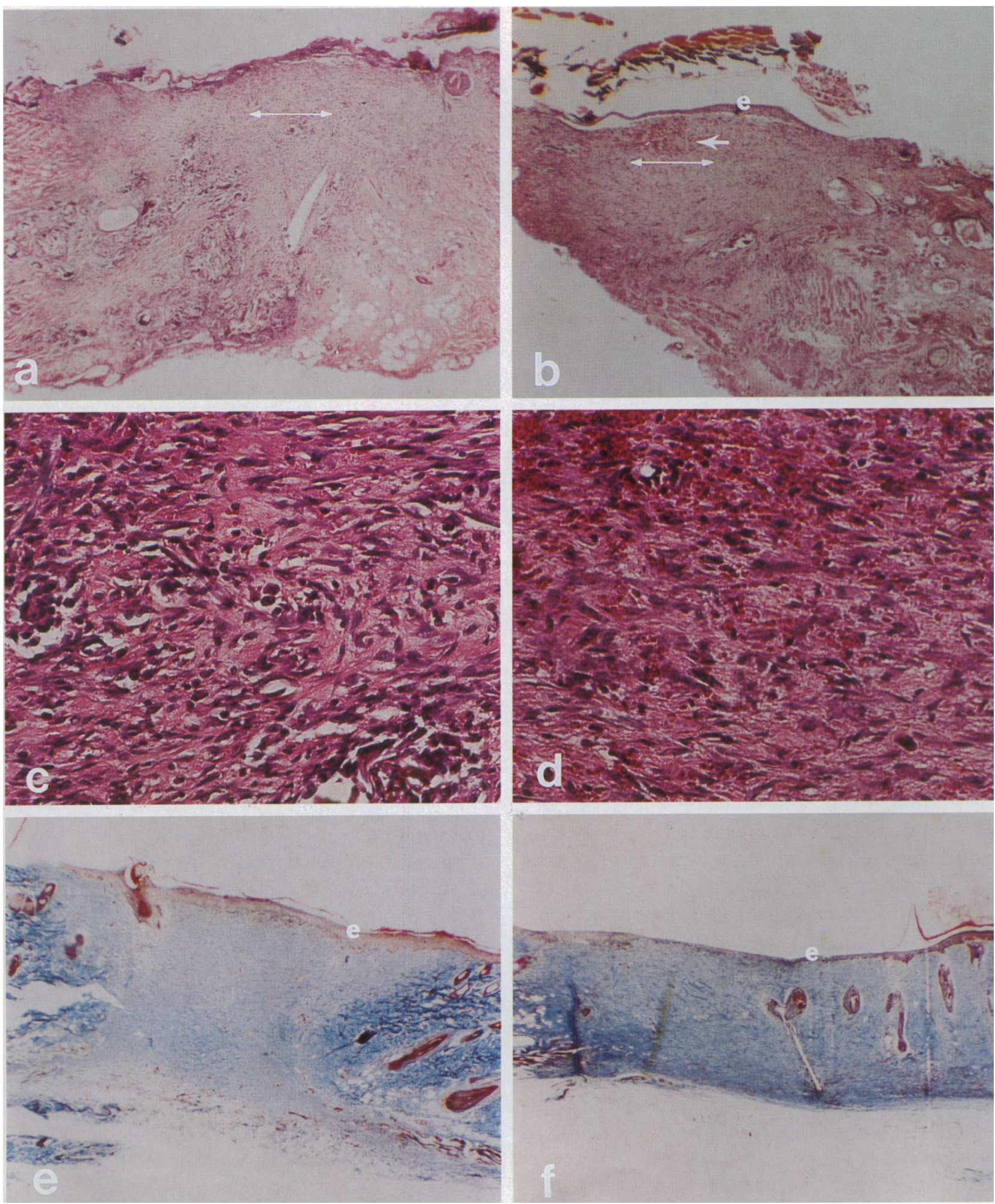

Figure $3 \mathrm{~A}$, Hematoxylin and eosin staining of control wound at 7 days shows presence of epidermis over granulating wound (original magnification $\times 25$ ). B, Day 7 flap wound stained with hematoxylin and eosin shows an area of prominent cellularity (arrows) just below the epidermis (e) (x25). C. Higher magnification of normal granulation tissue in control wounds noted by arrows in A (x125). D. Higher magnification of the area noted by arrows in $\mathbf{B}$ showing significant erythrocyte and cellular debris just below the epidermis in a flap wound (x125). E, Trichrome staining of a day 9 control wound shows a normal full-thickness architecture, as noted by the full layer of epidermis over the healing wound which contains abundant lightly staining blue material ( $\times 25)$. F, A corresponding day 9 flap wound shows a thinning of all layers from the epidermis $(e)$ down to the panniculus carnosus. Note the paucity of cell layers in the epidermis (x25). 


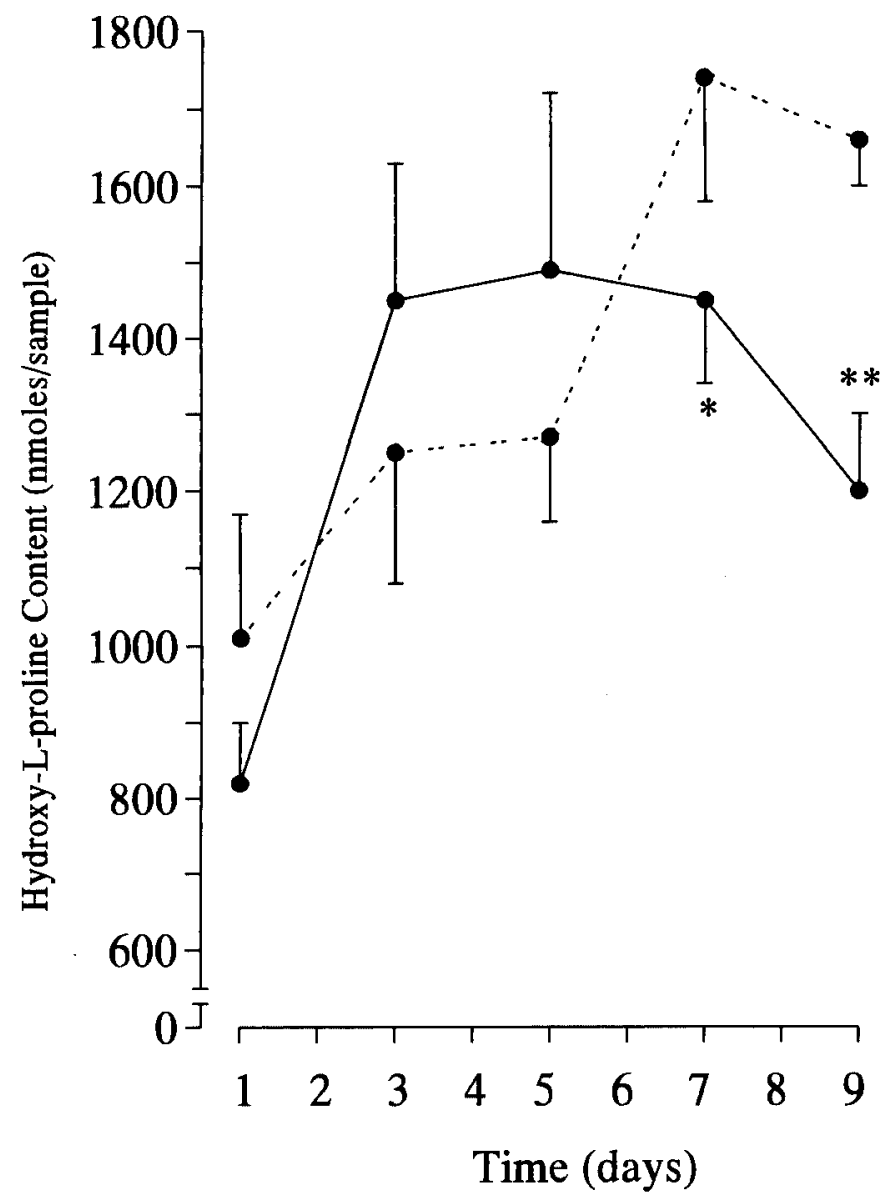

Figure 4 Total collagen as measured by 4 -Hyp values in flap wounds compared with controls. (Solid line represents flap wounds, dashed line represents control wounds). Wound 4-Hyp levels fall by day 7 and are $27 \%$ less than control wounds by day 9 ( $p<$ $0.05, " p<0.001)$. Values are the mean \pm standard deviation.

then rose to the 1400 to $1500 \mathrm{nmol}$ level and remained there through day 7 before dropping to $1203 \mathrm{nmol}$ at 9 days. The control wounds increased on days 7 and 9 to $1746 \mathrm{nmol}$ and $1658 \mathrm{nmol}$, respectively. The increase in 4-Hyp content of control wounds was statistically significant on days 7 and 9 compared with days 1 through $5_{\alpha}(p<0.005)$. In contrast, the dramatic decrease in flap wound 4-Hyp was statistically significant on day 9 as compared with days 3 through $7(p<$ 0.05 ). The total collagen content as measured by $4-$ Hyp was significantly lower in flap wounds compared with controls on days $7(p<0.05)$ and $9(p<0.001)$.

Relative collagen synthesis was measured in vitro as the percent collagen synthesized relative to noncollagen protein synthesis (Table 1). Both control and flap wound groups initially have biosynthetic levels below $2 \%$. Flap wound collagen synthesis gradually increased but did not peak until day $7(4.88 \%)$ then fell sharply to below $2 \%$ by day 9 . The control wounds increased more rapidly and peaked by day 5

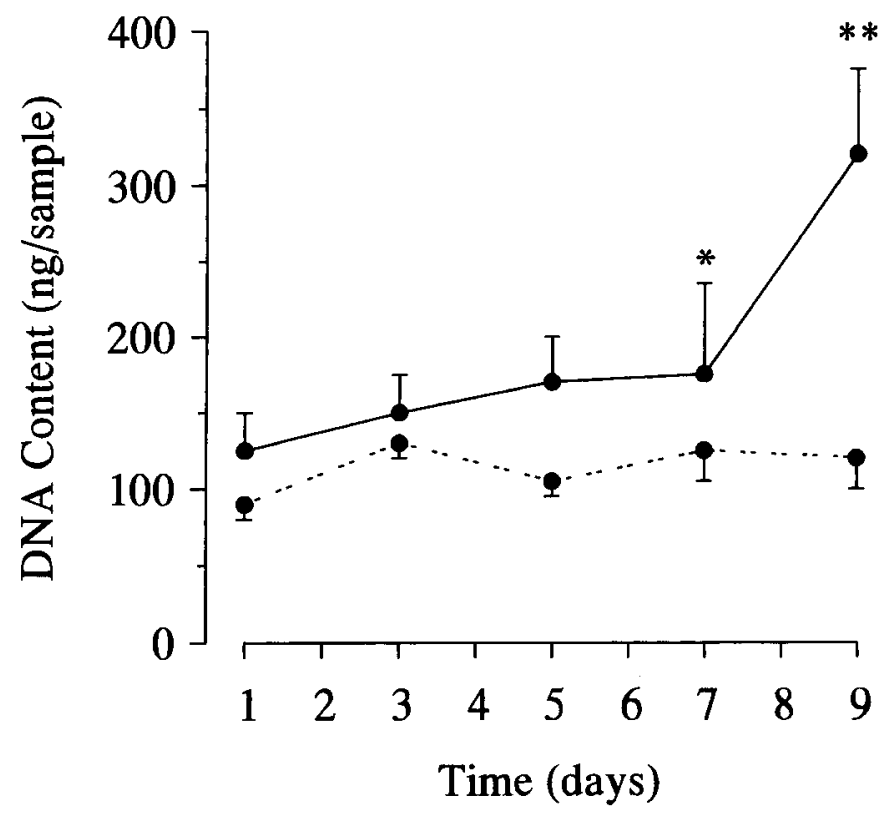

Figure 5 DNA content of excisional wounds expressed as nanograms/sample. (Solid line represents flap wounds, dashed line represents control wounds). DNA content in flap wounds show a progressive increase up through day 7 . with a rapid rise by day 9 not seen in control wounds ( $p<0.05, " p<0.001)$. Values are the mean \pm standard deviation.

(4.48\%) then declined slowly over days 7 through 9 but remained above $2 \%(2.37 \%)$. With two-way analysis of variance, no statistically significant difference was observed in collagen synthesis between flap and controls wounds at any time.

Absolute collagen synthesis was determined as radioactivity incorporated per microgram of DNA. Table 1 shows the values from day 1 through 9 as described between flap and control wounds. Overall, both flap and control wounds had similar values on days 1 , 3 , and 7 , except for a transient decrease in day 5 flap wounds. Both flap and control wounds dropped off between days 7 and 9 , with flap wounds being significantly less than controls by day $9(p<0.05)$. Absolute noncollagen protein synthesis was calculated as radioactivity incorporated into noncollagenase digestible protein per nanogram of DNA (Table 1). Control wounds have similar values from days 1 to 7 , except for a transient drop at day $5(p<0.05)$ as compared with 1 and 7 . In the flap wounds, the highest values are at days 1 and 3 , with significant drops at days 5, 7 , and $9(p<0.01)$ as compared with day 1 . Overall, between groups, the absolute noncollagen protein synthesis is significantly less than controls by days 7 and 9 ( $p<0.005$ and $p<0.01$, respectively).

A steady rise in DNA content in flap wounds from day 1 through 9 was observed (Figure 5). In contrast, the control values were not significantly different be- 
Table 1. Collagen synthesis determined in flap and control wound tissue

\begin{tabular}{|c|c|c|c|}
\hline Time (day) & Relative CS (\%) & Absolute CS (cpm/ng DNA) & Absolute non-CPS (cpm/ng DNA) \\
\hline \multicolumn{4}{|l|}{ Flap wounds } \\
\hline 1 & $1.84 \pm 0.73$ & $0.08 \pm 0.04$ & $0.76 \pm 0.25$ \\
\hline 3 & $3.37 \pm 0.95$ & $0.15 \pm 0.04$ & $0.81 \pm 0.14$ \\
\hline 5 & $4.25 \pm 1.21$ & $0.06 \pm 0.04^{*}$ & $0.23 \pm 0.09$ \\
\hline 7 & $4.88 \pm 1.23$ & $0.11 \pm 0.04$ & $0.42 \pm 0.17^{*}$ \\
\hline 9 & $1.91 \pm 0.73$ & $0.02 \pm 0.01^{\dagger}$ & $0.21 \pm 0.13^{*}$ \\
\hline \multicolumn{4}{|l|}{ Controls } \\
\hline 1 & $1.63 \pm 0.24$ & $0.07 \pm 0.03$ & $0.79 \pm 0.30$ \\
\hline 3 & $4.06 \pm 0.37$ & $0.15 \pm 0.07$ & $0.65 \pm 0.22$ \\
\hline 5 & $4.48 \pm 1.20$ & $0.18 \pm 0.01$ & $0.38 \pm 0.35$ \\
\hline 7 & $3.18 \pm 0.45$ & $0.13 \pm 0.06$ & $0.75 \pm 0.25$ \\
\hline 9 & $2.37 \pm 0.44$ & $0.07 \pm 0.02$ & $0.50 \pm 0.05$ \\
\hline
\end{tabular}

Values represent the mean \pm standard deviation. CS, Collagen synthesis; CPS, collagen protein synthesis; cpm, counts per minute.

"Significantly different from control wounds; $p<0.005$.

+ Significantly different from control wounds; $p<0.05$.

tween time points. Significant differences between groups became apparent at day 7 , when flap wound DNA content exceeded controls $(p<0.05)$. The rise in flap wounds between day 7 and 9 became more noticeable and clearly exceeded control wounds $(p<0.001)$.

\section{DISCUSSION}

Data from these studies show a clear distinction between healing of normal skin and skin subjected to ischemia after flap surgery. We have previously shown the importance of preconditioning to enhance flap survival after the distal end is detached from its nutrient supply. ${ }^{12,14,15}$ This condition is an example of the stress response whereby protective mechanisms are upregulated to mitigate the detrimental effects of further injury ${ }^{20}$ such as ischemia. The effects are seen grossly by necrosis in the acute flap that has not undergone any ischemic preconditioning. Acute, necrotic flaps are associated with a high percentage of oxidized glutathione/total glutathione. ${ }^{15}$ After 1 week of preconditioning (before detachment of distal flap), the levels of glutathione reductase along with other protective antioxidant enzyme activities are increased. In addition, there are less effects of ischemia as measured by decreased oxidant enzyme activity, and free radical formation, and improved flap survival after distal detachment. 12,14,15,21,22 However, during the time of preconditioning, while protective enzyme systems are being upregulated, there is still a level of gradient ischemia which leads to necrosis in the distal flap after detachment. The highest percentage of oxidized glutathione occurs at day 4 after preconditioning. ${ }^{15}$ This is the day we chose to create our punch wounds within the flap, anticipating to obtain the most profound differences in healing between flap and control wounds. We would attribute these expected differences to a greater oxidant stress.

Previous data have shown a decreased blood flow known as gradient ischemia occurring in the bipedicle flap. ${ }^{6,23}$ The greater the distance along the flap from the dermal perforator, the greater the level of ischemia and thus the risk of necrosis after distal detachment of the flap. This finding has been shown with the use of radioactive microspheres correlated with direct measurement of flap necrosis..$^{23}$ Indeed in preliminary data with a laser Doppler flow technique, our flap wounds had significantly less blood flow than control wounds at early times after injury. Other animal models have studied the microcirculation directly up to the wounds by microscopic assessment of nutrient capillary blood flow. ${ }^{5,6,11}$ These models relied on the minimal thickness of skin on animal ears, whereas this condition would not be feasible in our model. We are currently undertaking various microangiopathic studies to further define the role of angiogenesis in our model of delayed wound healing.

Although several parameters of delayed wound healing were assessed in our model, none showed more dramatic differences then a persistence of the wound where the excision was created (Figures $1, B$, and 2). Our model clearly shows a statistically significant defect over time. Although the control wounds heal by 8 days, the flap wounds have a persistent wound through day 12 with no significant healing seen between days.

Histologic evaluation of samples obtained at days 7 and 9 combines a demonstration of abnormal architecture including decreased thickness of both dermis 
and epidermis, as well as a gross lack of new collagen formation. The specific types of cells cannot be accurately distinguished by this method. One can, however, distinguish the presence of new collagen deposition in the control wounds by the light blue staining with trichrome. Our model grossly shows a wider gap of granulation tissue present between margins, correlating with the larger wound area measurements.

Hydroxyproline assessment showed an overall decrease in deposition of new collagen in excisional flap wounds after 1 week. The 4-Hyp measured at 9 days correlates well with both the histologic differences seen in relation to lack of new collagen deposition, as well as decreased rate of wound closure. The early rise in 4-Hyp in flap wounds may be related to an increased breakdown of old collagen in the wound, as well as an initial stimulation of collagen synthesis in the flap wounds which exceeded control wounds. However, by day 5 , no significant difference was found in the 4-Hyp content between control and flap wounds. Actual degradation of collagen later along the time course could result from enhanced proteolytic activity in the flaps leading to the $27 \%$ decrease in flap wound 4-Hyp by day 9 .

Of note, in vitro collagen synthesis is only marginally affected by this gradient ischemia. Previous studies have suggested that the presence of oxidants, or free radicals, stimulates collagen synthesis. ${ }^{24,25}$ This stimulation can be seen in our model where there is an initial increase in flap wound collagen synthesis on day 1 as compared with controls, although not statistically significant. The lack of a significant rise in collagen synthesis in the flap wounds as compared with controls may result from several experimental conditions.

The determination of an exact in vivo rate of collagen biosynthesis is difficult to obtain experimentally. Consequently, an in vitro method was selected for these studies. ${ }^{17}$ In this method, the wound tissue is removed from the animal and incubated for 4 hours under normoxic conditions with abundant synthetic substrates. Therefore, this method determines the capacity for the cells to synthesize collagen given this in vitro environment, which differs significantly from the wound environment. It is possible that in a hypoxic environment, with potentially limiting substrates or co-factors, the total collagen synthesized and secreted by the cells may be significantly less. Despite these limitations, this method allows one to ascertain the potential collagen synthetic rate, which in this study was not significantly different between ischemic and control excisional wounds.

Other potential experimental variables may have contributed to these findings. Cohen, Moore, and Diegelmann ${ }^{17}$ found a statistically significant increase in collagen synthesis in wound tissue compared with intact normal skin. In addition, they also noted a significantly higher level of collagen synthesis in the center of the wound (granulation tissue) as compared with wound margins. In our model, we have shown a persistent failure of the flap wounds to heal. By taking a $6 \mathrm{~mm}$ excision of the wound, one would be sampling a larger portion of normal skin in controls where the actual area of wound is significantly smaller. On the basis of previous data, the relative collagen synthesis from control wounds in a $6 \mathrm{~mm}$ sample should more closely resemble normal skin. For example, we might anticipate a higher relative collagen synthesis in our control wounds as compared with flap wounds if we were to take a smaller excision around the punch wound which contained only granulation tissue (less normal skin) to perform collagen synthesis determinations.

Another possibility which would explain the lack of significant difference in relative collagen synthesis is a prolonged inflammatory phase in the flap wounds. Studies support inflammatory cells (primarily macrophages) and their cytokines stimulating fibroplasia, collagen synthesis, and angiogenesis. ${ }^{10,26}$ Conversely, it is possible that the inflammatory response with resulting protease activity may degrade procollagen, leading to a lower determination of collagen synthetic rate in flap wounds than expected. In preliminary studies with myeloperoxidase as an enzymatic marker for neutrophil content, we have reported a significant prolongation of the time neutrophils are present within the ischemic wounds. ${ }^{27}$ This finding would also be consistent with the observation in the current study of a significant elevation in DNA, possibly from neutrophils, within the ischemic wounds after day 5 (Figure 5).

Finally, on the basis of the literature that supports stimulation of collagen synthesis by an anaerobic environment and free radicals in ischemic wounds, ${ }^{24,25,28}$ we should expect increased collagen synthesis in the flap wounds. However, other literature supports increased collagen synthesis with higher oxygen tension and angiogenesis, ${ }^{7,8}$ and thus we might expect decreased collagen synthesis in our ischemic flap wounds. Overall, our data shows a decrease in both absolute collagen and noncollagen protein synthesis by day 9 in flap wounds versus controls. Because relative collagen synthesis is a ratio between collagen and noncollagen protein synthesis, a similar decrease in both would explain no change in the ratio, thus no difference in relative collagen synthesis. 
In summary, these data indicate that excisional wounds in our ischemic wound model are significantly delayed in their time to closure. This delay is associated with a significant failure to accumulate collagen, although the cells within the wound have the capacity to synthesize collagen at a rate comparable with control wound cells. Therefore, our findings may represent a combination of wound environmental factors which increase collagen turnover by enhancing the proteolytic environment and possibly prevent the cells from expressing their potential to synthesize collagen and other proteins.

\section{ACKNOWLEDGEMENTS}

We thank Belinda Adamson, MEd, and Judy Thul for their technical assistance; William Kuzon, MD, $\mathrm{PhD}$, for statistical analysis; Robert Gilmont, $\mathrm{PhD}$, for insight with data interpretation and experimental design; and Michael Welsh, PhD, for assistance with histologic evaluation. These studies were supported by the Plastic Surgery Educational Foundation and the Department of Veterans Affairs. A portion of these results was presented at the Surgical Forum, Chicago, Illinois, October 1994.

\section{REFERENCES}

1. Van Den Brenk HAS, Sharpington C, Orton O, Stone M. Effects of X-radiation on growth and function of the repair blastema (granulation tissue). Int J Radial Biol 1974;25:277-89.

2. Brennan SS, Leaper DJ. The effect of antiseptics on the healing wound: a study using the rabbit ear chamber. Br J Surg 1985;72:780-2.

3. Cliff WJ. The behavior of macrophages labelled with colloidal carbon during wound healing in rabbit ear chambers. Quart $\mathrm{J}$ Exp Physiol 1966;51:112-9.

4. Eriksson E, Boykin JV, Pittman RN. Method for in vivo microcirculation of the hairless mouse ear. Microvasc Res 1980;19:3749.

5. Barker JH, Hammersen F, Bondar I, Uhl E, Galla TJ, Menger MD, Messmer K. The hairless mouse ear for in vivo studies of skin microcirculation. Plast Reconstr Surg 1989;83:948-59.

6. Barker JH, Hammersen F, Bondar I, Galla TJ, Menger MD, Gross W, Messmer K. Direct monitoring of nutrient blood flow in a failing skin flap model. Plast Reconstr Surg 1989;84:30313 .

7. Hunt TK, Pai MP. Effect of varying ambient oxygen tension on wound metabolism and collagen synthesis. Surg Gynecol Obstet $1972 ; 135: 561-7$.

8. Niinikoski J, Hunt TK, Dunphy JE. Oxygen supply in healing tissue. Am J Surg 1972;123:247-52.

9. Knighton DR, Hunt TK, Thakral KK, Goodson WH. Role of plate- lets and fibrin in the healing sequence. Ann Surg 1982;196:37988.

10. Hunt TK, Knighton DR, Thakral KK, Goodson WH, Andrews WS. Studies on inflammation and wound healing: angiogenesis and collagen synthesis stimulated in vivo by resident and activated wound macrophages. Surgery 1984;96:48-54.

11. Ahn ST, Mustoe TA. Effects of ischemia on ulcer wound healing: a new model in the rabbit ear. Ann Plast Surg 1990;24:1722 .

12. Rees R, Punch J, Shaheen K, Cashmer B, Guice K, Smith D The stress response in the skin: the role of neutrophil products in preconditioning. Plast Reconstr Surg 1993;92:110-6.

13. McFarlene RM, Heagy FC, Radin S, Aust JC, Wermuth RE. A study of the delay phenomenon in experimental pedicle flaps. Plast Reconstr Surg 1965;35:245-62.

14. Rees R, Smith D, Li TD, Cashmer B, Garner W, Punch J, Smith DJ. The role of xanthine oxidase and xanthine dehydrogenase in skin ischemia. J Surg Res 1994;56:162-7.

15. Rees RS, Smith DJ Jr, Adamson B, Im M, Hinshaw D. Oxidant stress: the role of the glutathione redox cycle in skin pre-conditioning. J Surg Res [In press].

16. Lindblad WJ, Diegelmann RF. Quantitation of hydroxyproline isomers in acid hydrolysates by high performance liquid chromatography. Anal Biochem 1984;138:390-5.

17. Cohen IK, Moore CD, Diegelmann RF. Onset and localization of collagen synthesis during wound healing in open rat skin wounds. Proc Soc Exp Biol Med 1979;160:458-62.

18. Diegelmann RF, Peterkofsky B. Collagen biosynthesis during connective tissue development in chick embryo. Dev Biol 1972;28:443-53.

19. Labarca C, Paigen K. A simple, rapid, and sensitive DNA assay procedure. Anal Biochem 1980;138:344-52.

20. Welsh WJ. How cells respond to stress. Sci Am 1993:56-64.

21. Rees R, Hinshaw D, Cashmer B, Im MJ, Smith DJ. Experimental evidence that oxidative stress from delay upregulates antioxidant defenses in ischemic skin. Surg Forum 1991;42: 648-9.

22. Rees RS, Kingman G, Cashmer BA, Reaves C, Gilmont R, Welsh M, Smith DJ. DT diaphorase: increased enzyme activity in oxidant stress in skin. J Surg Res 1994:56:326-30.

23. Bean D, Rees RS, O'Leary JP, Lynch JB. Comparison of blood flow and cellular function in ischemic skin flaps. J Surg Res 1984;37:69-74.

24. Hussain MZ, Bhatnagar RS. Involvement of superoxide in the paraquat-induced enhancement of lung collagen synthesis in organ culture. Biochem Biophys Res Comm 1979;89:71-6.

25. Hussain MZ, Watson JA, Bhatnagar RS. Increased prolyl hydroxylase activity and collagen synthesis in hepatocyte cultures exposed to superoxide. Hepatology 1987;7:502-7

26. Pierce GF, Brown D, Mustoe TA. Quantitative analysis of inflammatory cell influx, procollagen tyve I synthesis, and collagen cross-linking in incisional wounds: influence of PDGFBB and TGF- $\beta$ therapy. J Lab Clin Med 1991;117:373-82.

27. Schwarz DA, Finklestein S, Rees RS, Lindblad WJ. Acute inflammation is associated with metalloproteinase activity in a rat model of delayed wound healing |Abstract|. Wounu RFP RFG 1995;3:101.

28. Hunt TK, Conolly WB, Aronson SB, Goldstein P. Anaerobic metabolism and wound healing: an hypothesis for the initiation and cessation of collagen synthesis in wounds. Am J Surg 1978;135:328-32. 